\title{
Carotenoids: nature's unique pigments for light and energy processing
}

\author{
Thomas A. Moore, Devens Gust and Ana L. Moore \\ Department of Chemistry and Biochemistry and Center for the Study of Early Events in Photosynthesis, \\ Arizona State University, Tempe, AZ 85287 USA
}

\begin{abstract}
Covalently linked carotenoid-porphyrin (C-P) dyads that mimic the photochemistry of carotenoids in photosynthetic membranes have been synthesized. Singlet energy transfer (C to $P$ ), triplet energy transfer ( $P$ to $C$ ), and quenching of $P^{*}$ by electron transfer from $C$ to $P^{*}$, have been observed and the structural requirements for these processes defined. A monolayer system has been developed in which a carotenoid bearing a terminal amino group is photoelectrochemically active. This demonstrates that carotenoid pigments could act as blue light photoreceptor pigments. A carotenoporphyrin for use as an imaging agent for tumor tissue detection has been synthesized and tested in vivo. This compound incorportates carotenoid photoprotection against singlet oxygen and is therefore not phototoxic.
\end{abstract}

\section{INTRODUCTION}

Carotenoid pigments are ubiquitous in the photosynthetic membranes of plants and bacteria where they are involved in the collection of sunlight for photosynthetic work, the dissipation of excited triplet-state energy, and the regulation of singlet energy flow to the photosynthetic reaction centers (refs. 1,2). Recent evidence also implicates these pigments as light receptors for photosensory processes in plants (ref. 3). Additionally, carotenoid pigments are found throughout the plant and animal kingdoms where they provide cryptic coloration.

Although the observation of these functions in vivo suggests a rich photochemical repertoire for carotenoid pigments, in solution they display disappointingly little photochemistry, do not undergo intersystem crossing to their triplet-states, do not phosphoresce and are only very weakly fluorescent (ref. 4). Moreover, the intense absorption that gives carotenoids a characteristic yellow to orange color involves an electronic transition to the second excited singletstate. The lowest excited singlet-state has a very small extinction coefficient and is not easily observed by conventional spectroscopic methods even though it is a level from which interesting photochemistry would be expected to occur. However, carotenoids in solution do display pigment-pigment interactions that give rise to large changes in the energy level of the second excited singlet-state with concomitant shifts of the absorption spectrum to wavelengths that mimic some of the colors found in vivo.

In contrast to the paucity of photochemistry displayed by individual carotenoid pigments, their interaction with an attendant chromophore unleashes a rich spectroscopy and photochemistry. This can be achieved by covalently linking the carotenoid to another pigment. Because the photochemical functions of carotenoids in photosynthesis involve carotenoid-chlorophyll interactions, we have chosen to model these functions using a series of covalently linked carotenoid-porphyrin (C-P) dyads. Tetraarylporphyrins were chosen because they are good models for chlorophyll, their electronic energy levels and redox potentials can be systematically varied by chemical derivatization, and their linkage to other pigments can be varied to control the extent of electronic interaction (ref. 5).

Singlet energy transfer $(C$ to $P)$, triplet energy transfer $(P$ to $C)$, and $C$ quenching of $P^{*}$ by electron transfer from $C$ to $\mathrm{P}^{*}$ have been observed in a series of carotenoporphyrins. A knowledge of the photochemistry of these compounds has important consequences for understanding the functions of carotenoids in living organisms. For example, the structures and interactions that give rise to carotenoid photochemistry can be used to make predictions for in vivo structures of carotenoid-chlorophyll binding proteins. Information about how chromophore-chromophore interactions specify certain photochemical processes involving carotenoids can be used to design photoactive drugs which may be useful in humans (ref. 6). In this review, some of the carotenoporphyrin dyads that display the photochemistry mentioned above will be presented and the relationship between their structure and photochemical function will be discussed. The work reporting $C$ quenching of $P^{*}$ has recently been published and is not reviewed here (ref. 7 ).

\section{CAROTENOID-TO-PORPHYRIN SINGLET ENERGY TRANSFER}

It has been known for some time that light absorbed by carotenoids in photosynthetic membranes leads to photosynthetic activity, which implies that singlet energy transfer from carotenoid to chlorophyll occurs. However, 
the mechanism of this energy transfer is not obvious. In early work it was found that in solutions of carotene and chlorophyll, singlet energy transfer from the carotene to the chlorophyll could not be demonstrated. This result contrasts with those found for many other dye pairs, where energy transfer to the dye with the lowest energy excited singlet-state is easily observed and adequately described by the Förster dipole coupling mechanism.

The failure of carotenoid pigments in solution to participate in classical Förster-mediated energy transfer is now understood to be a consequence of the unusual electronic structure and photophysics of their excited states. As mentioned above, the characteristic carotenoid color is due to the strong absorption of blue light, which populates the second excited singlet-state $\left(S_{2}\right)$. The lowest excited singlet-state $\left(S_{1}\right)$ is electric dipole forbidden, does not contribute to the color of the carotenoid and is not detected in ordinary absorption studies. However, a variety of recent experiments have located $S_{1}$ in the red spectral range between ca. 630 and $710 \mathrm{~nm}$ for typical carotenoids of 10 and 11 conjugated double bonds (refs. 8-12). It is generally accepted that, upon excitation with blue light, $S_{2}$ relaxes in ca. 200 fs to populate $S_{1}$ which has a lifetime of ca. 10 to $40 \mathrm{ps}$ in different carotenoids (refs. 13,14). Many, if not most, carotenoids are only weakly fluorescent from $S_{2}, S_{1}$ or both states with quantum yields of ca. $1 \times 10^{-4}$ or less (refs. 15,16 ). In the case of $S_{2}$, the very low fluorescence quantum yield is a consequence of its ultrashort lifetime. The short lifetime of $S_{1}$ coupled with the optically forbidden nature of its transition to the ground-state render it almost nonfluorescent. These characteristics preclude efficientFörster energy transfer ${ }^{2}$ recause the dipole coupling mechanism requires that the donor have a probable fluorescent transition and therefore a reasonably strong transition dipole.

Porphyrin-porphyrin and carotenoporphyrin dyads 1 and 2 illustrate the consequences of the carotenoid electronic structure described above for singlet energy transfer to a porphyrin. Both dyads have the same linkage and therefore similar edge-to-edge separation, but very different singlet energy transfer properties. Porphyrin dyad 1 exhibits singlet energy transfer between the porphyrins with a yield of essentially $100 \%$, while in carotenoporphyrin 2 the yield of energy transfer is ca. $13 \%$ (refs. 17,18).
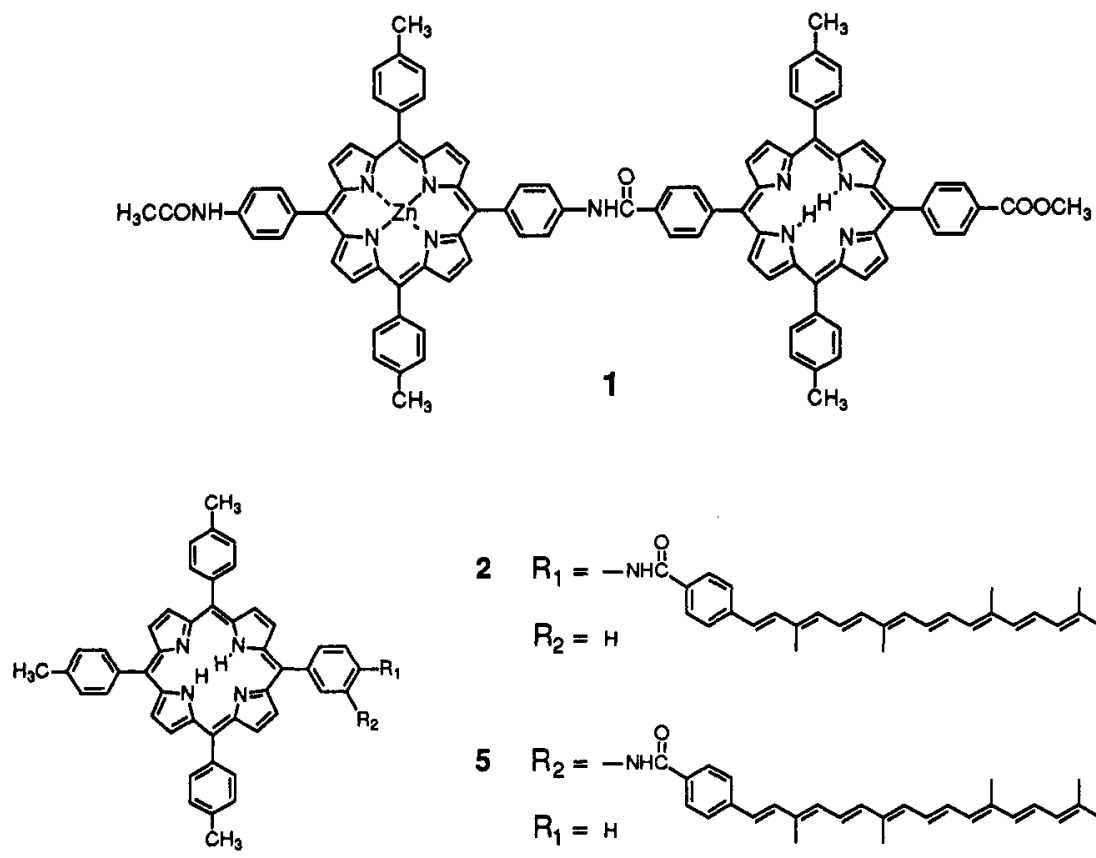

2

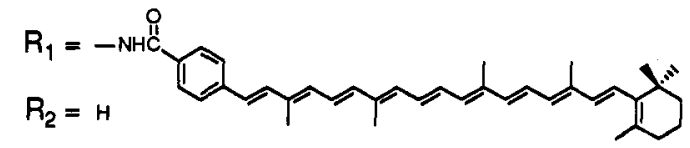

5

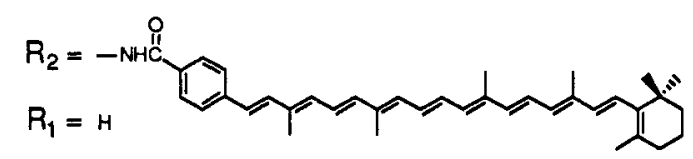

We have carried out a study of the dependence of carotenoid-to-porphyrin singlet energy transfer on the electronic structure of the linkage joining the two moieties and have concluded that in these model systems, energy transfer is mediated by a through-bond interaction of the chromophores (ref. 18). Although some contribution from a Forster-type dipole coupling mechanism cannot be ruled out absolutely, specific molecular conformations would have to be arbitrarily selected to support that view. The through-bond interaction referred to here is a type of electron exchange interaction and has specific structural requirements. Most importantly, electron exchange interactions require that the distance between the chromophores be short enough for orbital overlap to occur. For direct orbital overlap to be effective, the chromophores must be in van der Waals contact. For unlinked species in solution this requires a collision and therefore ties the rate of the process to the rate of diffusion. For linked species direct orbital overlap can occur through conformations in which the $\pi$-systems of the two chromophores are in contact. If overlap is indirect, involving orbitals of the intervening linkage or intervening material such as solvent or protein, the interaction is known as through-bond or super-exchange. In c trast to the electron exchange mechanism, the dipole coupling mechanism occurs through space and does not requi orbital contact. It is now clear why carotenoids are 
not efficient singlet energy donors in solution. Their $S_{1}$ lifetime of only a few tens of $p s$ is so short that collisions with prospective energy acceptors (porphyrins or chlorophylls) do not occur on that time scale.

The finding that electron exchange is necessary to mediate singlet energy transfer from carotenoid pigments has important consequences for the evolution of chlorophyll-carotenoid binding proteins for photosynthesis (ref. 19). In order for the carotenoid to act as an antenna by transferring singlet excitation energy to the chlorophyll system, the two pigments must be protein-bound so that they are in van der Waals contact with each other.

The crucial nature of the interaction required for successful energy transfer is illustrated by the result for dyads 3 and 4 in which a carotenoid is linked to a pyropheophorbide derivative (Ppd) (refs. 20,21). Inspection of the linkages reveals saturated carbons separating the chromophores in 3 and the partial conjugation between them provided by the amide linkage in 4.
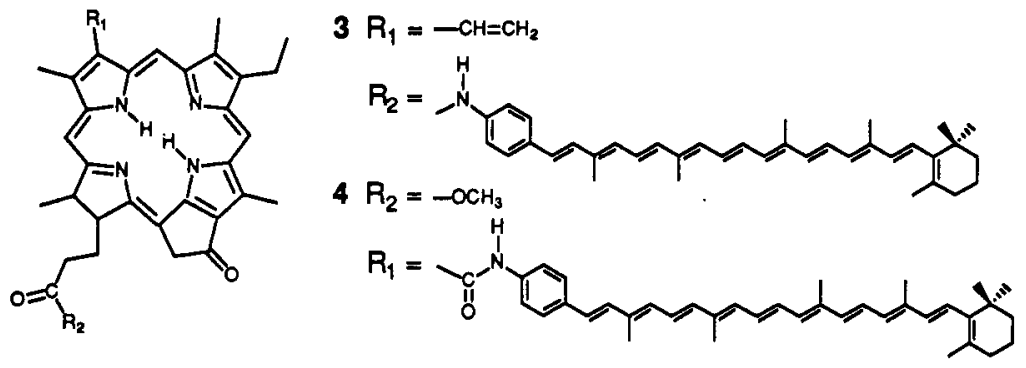

Singlet energy transfer from the carotenoid to Ppd in these compounds has been measured by both steady-state and time-resolved methods. In the case of 3, energy transfer measured by carotenoid-sensitized Ppd fluorescence (steady-state experiment) is less than ca. $5 \%$. Consistent with this observation, time-resolved experiments showed that the carotenoid $S_{1}$ singlet lifetime ( $16 \mathrm{ps}$ ) was not quenched by the attached Ppd. By contrast, the carotenoid $S_{1}$ lifetime of dyad 4 was found to be quenched to $7 \mathrm{ps}$. Energy transfer was confirmed to be the quenching mechanism by the agreement between the 55\% efficiency calculated from the dynamic data and the 53\% measured in steady-state experiments. Our interpretation of these results is that the saturated carbons in the linkage of $\mathbf{3}$ effectively isolate the two chromophores from $\pi$-orbital contact, thereby preventing the electron exchange interactions necessary to mediate efficient singlet energy transfer. The partial conjugation of the amide linkage in $\mathbf{4}$ provides a mechanism for electron exchange interactions between the chromophores, and therefore reasonably efficient energy transfer.

The time-resolved experiments strongly implicate the carotenoid $S_{1}$ state as the donor because the $7 \mathrm{ps}$ time constant for $S_{1}$ quenching matches the $7 \mathrm{ps}$ rise time for the Ppd singlet species. The carotenoid $S_{2}$ state lives, at most, several hundred fs and therefore could not contribute to events on the ps time scale. Regarding the thermodynamics of the process, the $S_{1}$ energy level of the carotenoid moiety of 3 and 4 can be calculated from its $S_{1}$ lifetime and the energy gaplaw (ref. 12) to be essentially isoenergetic with that of the Ppd. Thus, the energy transfer is thermodynamically allowed. However, near degeneracy of the excited singlet levels of chlorophyll and carotenoid species could lead to interesting experimental energy transfer results. If the chlorophyll and carotenoid excited singlet species are in equilibrium, the net energy transfer efficiency would depend on their individual singlet lifetimes. In the absence of additional rapid photochemical processes that quench the chlorophyll singlet, which could be the case in isolated particles or model systems, energy transfer efficiency would be less than optimal. On the other hand, fast energy transfer or electron transfer from the chlorophyll singlet to an additional species (such as a neighboring chlorophyll), which would be the case in intact photosynthetic membranes, would result in an efficient net flux of singlet energy transfer from the carotenoid. It seems safe to conclude that for most carotenoid pigments $S_{1}$ is sufficiently energetic to act as the donor species in chlorophyll-based photosynthesis and even more so in bacteriochlorophyll-based systems.

\section{PORPHYRIN-TO-CAROTENOID TRIPLETENERGY TRANSFER}

Because there are no large transition dipoles associated with the ground-state to triplet-state transitions in organic chromophores, triplet-triplet energy transfer is mediated by electron exchange interactions between the pigments. In the case of the carotenoids and tetrapyrroles considered here, the same general structural requirement for orbital overlap that was found for singlet energy transfer, either direct or via through-bond coupling, has been found for triplet energy transfer (ref. 18).

Oxygen-evolving photosynthetic organisms require photoprotection from singlet oxygen for their survival. Carotenoid pigments are implicated in photoprotection in two ways, illustrated by reactions 1 and 2 .

$$
\mathrm{C}+{ }^{1} \mathrm{O}_{2} \longrightarrow{ }^{3} \mathrm{C}+{ }^{3} \mathrm{O}_{2} \text { (ground-state oxygen) }
$$




$$
\mathrm{C}+{ }^{3} \mathrm{Chl} \longrightarrow{ }^{3} \mathrm{C}+\mathrm{Chl} \text { (ground-state chlorophyll) }
$$

In reaction (1), singlet oxygen is physically quenched by the carotenoid to yield the triplet carotenoid species, which decays harmlessly to the ground-state, and ground-state dioxygen. Many studies have shown that carotenoids of ca. nine conjugated double bonds or more are efficient quenchers of singlet oxygen. It is reasonable to expect this mechanism in tissues or organelles in which the carotenoid concentration is very high. However, a serious limitation of this strategy is that the carotenoid must compete with other components of the cell which are highly reactive with singlet oxygen. Because singlet oxygen reacts with many biochemical compounds including unsaturated lipids, various organic cofactors and certain amino acids, it is difficult to imagine that photoprotection by random diffusion would be universally successful. In essence, this mechanism requires that each compound susceptible to attack by singlet oxygen be surrounded by a shield of carotenoids.

Reaction (2) represents the quenching of the sensitizer triplet chlorophyll before it can transfer energy to dioxygen to form singlet oxygen. Although this mechanism also involves a competition, this time between the carotenoid and dioxygen for the triplet chlorophyll, the nature of the photoprotection is very different from that in reaction 1 . Only the source of the singlet oxygen need be quenched. The necessary chlorophyll-carotenoid energy transfer rate can be ensured by the structure of the pigment-bearing protein.

In order to determine the structural and photophysical parameters required for successful photoprotection by reaction (2), the competition between dioxygen and carotenoid for the sensitizer triplet energy has been studied in the isomeric carotenoporphyrin dyads 2 and 5 (ref. 18). The relationship between the porphyrin and carotenoid moieties at the meso-aryl group in the linkage is para in 2 and meta in 5 . Because triplet energy transfer is mediated by throughbond electron exchange interactions, and such interactions are weaker when the coupled groups are meta to one another, the triplet energy transfer rate constant is lower for 5 than for 2.

2: $\mathrm{C}$ para ${ }^{3} \mathrm{P} \longrightarrow{ }^{3} \mathrm{C}$ para $\mathrm{P} k>1 \times 10^{8} \mathrm{~s}^{-1}$

5: $\mathrm{C}$ meta ${ }^{3} \mathrm{P} \longrightarrow{ }^{3} \mathrm{C}$ meta $\mathrm{Pk}=2 \times 10^{7} \mathrm{~s}^{-1}$

The rate constants shown above were determined in toluene solution by laser flash spectroscopy. The amount of singlet oxygen produced by each compound was measured by direct observation of the singlet oxygen emission at $1.27 \mu \mathrm{m}$. The oxygen-sensitization reaction, $\mathrm{C}-{ }^{3} \mathrm{P}+{ }^{3} \mathrm{O}_{2} \longrightarrow \mathrm{C}-\mathrm{P}+{ }^{1} \mathrm{O}_{2}$, has a pseudo first order rate constant of ca. $1 \times 10^{7} \mathrm{~s}^{-1}$ at $10 \mathrm{mM}$ dioxygen concentration. When the yield of singlet oxygen $(\Phi \Delta)$ from a free base tetraarylporphyrin was used as a reference, the yield for the meta-linked carotenoporphyrin 5 was 0.3 and no singlet oxygen could be detected from the para-linked isomer 2 . Based on the rate constants given above, the calculated singlet oxygen yield for the meta isomer is 0.33 , in excellent agreement with the observed yield.

This experiment defines the kinetic constraints on the triplet energy transfer process necessary to suppress completely the formation of singlet oxygen, and permits several observations. The triplet energy transfer must be very fast to suppress singlet oxygen formation completely. Interestingly, for 5 the agreement between the calculated yield and the observed yield means that the attendant carotenoid did not trap an appreciable fraction of the singlet oxygen sensitized by the attached porphyrin. This implies that singlet oxygen escape from the collision complex producing it is highly probable even with a powerful quencher in close proximity. Apparently, evolutionary pressure has forced photosynthetic organisms to avoid the sensitization altogether. In reaction centers of Rhodobacter sphaeroides the bacteriochlorophyll special pair triplet is quenched by energy transfer to the carotenoid with a rate constant of $>10^{8} \mathrm{~s}^{-1}$ (ref. 22).

The triplet energy transfer in $R b$. sphaeroides is not a simple process. The crystal structure of the reaction center presents a paradox in that the carotenoid is bound in van der Waals contact with an accessory bacteriochlorophyll on the $B$ polypeptide branch and not in direct contact with the most likely site of triplet formation, the special pair (ref. 23). This arrangement suggests that the rapid quenching observed is a two-step process involving, first, triplet energy transfer from the special pair bacteriochlorophylls to the accessory bacteriochlorophyll, and then transfer to the carotenoid.

In order to explore the structural and dynamic requirements for photoprotection involving a two-step triplet energy transfer relay, we have synthesized molecular triad 6 comprising a carotenoid linked to a porphyrin that is in tum linked to a pyropheophorbide derivative (C-P-Ppd) (ref. 24).

Excitation of either the P or Ppd chromophore of an acetone solution of 6 populates the first excited singlet-state of the Ppd moiety, which has the lowest excited singlet level in the triad and therefore accepts singlet energy from P*. Intersystem crossing yields triplet Ppd which is an excellent sensitizer of singlet oxygen, with $\Phi \Delta \mathrm{ca} .0 .8$. The dyad P-Ppd also sensitizes singlet oxygen with the same high quantum yield. Attaching the carotenoid pigment to form 6 reduces the quantum yield of singlet oxygen to 0.36 . As was the case for 5 , these results reflect the competition for the triplet Ppd species between dioxygen and intramolecular energy transfer. Intramolecular triplet energy transfer in 6 is a two-step relay process in which the first step involves transfer from C-P- ${ }^{3} \mathrm{Ppd}$ to form C- ${ }^{3} \mathrm{P}-\mathrm{Ppd}$, and the second step forms ${ }^{3} \mathrm{C}$-P-Ppd. Triplet transfer from C-P- ${ }^{3} \mathrm{Ppd}$ to yield ${ }^{3} \mathrm{C}-\mathrm{P}-\mathrm{Ppd}$ in 6 is slow, however, because the step from 


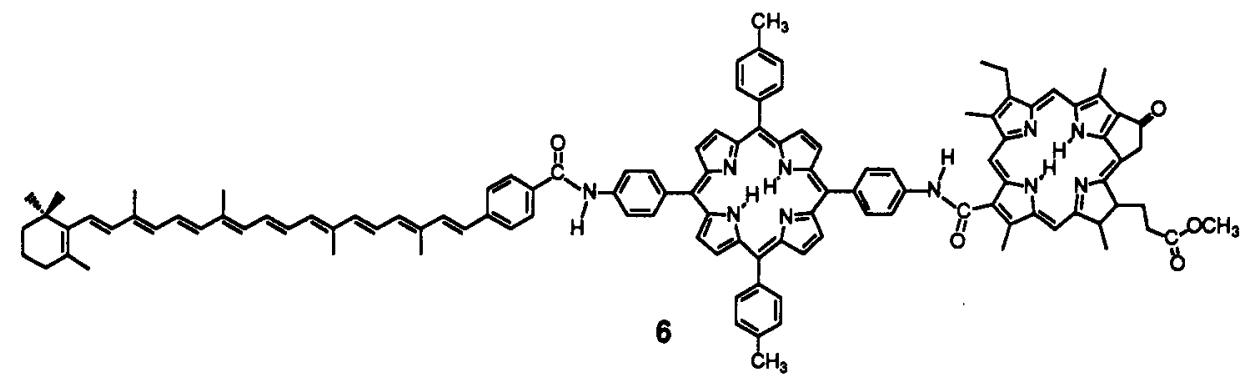

${ }^{3} \mathrm{Ppd}$ to the intermediate ${ }^{3} \mathrm{P}$ is endergonic by $\mathrm{ca} 0.11 \mathrm{eV}$. The activated, rate-limiting step slows the triplet transfer process, and allows significant sensitization of singlet oxygen. The triplet transfer rate constant is ca. $3 \times 10^{6} \mathrm{~s}^{-1}$ and the pseudo first order rate constant for dioxygen quenching in air-saturated acetone is ca. $5 \times 10^{6} \mathrm{~s}^{-1}$. These rate constants and $\Phi \Delta$ for Ppd give $\Phi \Delta=0.30$ for 6, in satisfactory agreement with the measured value, 0.36 .

One consequence of the activated triplet energy transfer step in 6 is that the overall process is strongly temperature dependent. At 77K in a glassy solution there is no observable triplet energy transfer to the carotenoid. This is also the case for the natural reaction center mentioned above, but the activation energy is much lower so that the transfer slows significantly only below ca. 50K. In dyads such as 2 and 5 triplet energy transfer is not temperature dependent (at least above 77K). It is relatively facile to change the activation barrier height in the triad. Insertion of a zinc ion in the porphyrin moiety of 6 increases the activation energy by $0.15 \mathrm{eV}$ due to the higher energy of ${ }^{3} \mathrm{PZn}$. In this compound triplet energy transfer is too slow to compete with singlet oxygen sensitization and this results in negligible photoprotection by the carotenoid.

These experiments have established several principles regarding multistep triplet energy transfer and the suppression of singlet oxygen. To be effective, the photoprotective mechanism must feature rapid triplet energy transfer from the potential sensitizer to the carotenoid. Close proximity of the carotenoid to the sensitizer without energy transfer provides essentially no suppression of singlet oxygen. One carotenoid could protect several tetrapyrrole pigments if these were individually exchange-coupled to the carotenoid. If the tetrapyrroles are coupled to each other and there are no insurmountable thermodynamic barriers, then only one of them need be coupled to the carotenoid. From the results for 6 , it is clear that a relay can provide a triplet energy conduit to a proximal carotenoid.

\section{ENERGY OF THE CAROTENOID TRIPLET-STATE}

The essential element of photoprotection is that ${ }^{3} \mathrm{C}$ does not sensitize an appreciable quantity of singlet oxygen. There is nothing in the electronic structure, spin statistics, or steric factors of the carotenoid - dioxygen interaction that can account for this observation. Indeed, ${ }^{3} \mathrm{C}$ is quenched at near the diffusion limit by dioxygen to yield the two groundstate species and, as mentioned above, singlet oxygen is strongly quenched by ground-state $\mathrm{C}$ to yield ${ }^{3} \mathrm{C}$. This leaves thermodynamics as the only obvious explanation for the lack of sensitization of singlet oxygen by ${ }^{3} \mathrm{C}$. The energy of singlet oxygen is $0.98 \mathrm{eV}$ above the ground-state and therefore a potential sensitizer must have its lowest triplet energy near or above $1 \mathrm{eV}$. Quantitatively, singlet oxygen was not detected upon excitation of dyad 2 which, in our spectrometer, sets the lower limit for the quantum yield of sensitization at ca. $10^{-2}$ times that produced by a free base tetraarylporphyrin. If we assume that the quantum yield limit is due to the endergonic nature of the energy transfer process, which defines an activation barrier to singlet oxygen sensitization, then the carotenoid triplet energy is calculated to be $0.88 \mathrm{eV}$.

Experimentally, there are very few data in the literature regarding the energy of the carotenoid triplet-state. The most straightforward measurement would be to detect the phosphorescence and to recordits spectrum. This has proven futile for carotenoids in solution because the quantum yield of intersystem crossing is vanishingly small so that the triplet-state is not populated by excitation into the singlet manifold. Sensitization by a triplet donor ordinarily requires diffusion and collisions which are precluded in the frozen glassy matrix required to enhance phosphorescence. In order to overcome these obstacles, we have attempted to detect phosphorescence from the carotenoid moiety of carotenoporphyrin dyads such as $\mathbf{2}$ in which triplet sensitization is intramolecular and in which the triplet yield is greater than 50\% in frozen glasses. No signal was observed in the wavelength range 600 to $1300 \mathrm{~nm}$ (ref. 25). A traditional technique used in organic photochemistry to locate optically unobservable triplet-states is to perform a series of quenching experiments with triplet energy donors of descending energy. When the donor triplet energy is significantly lower than that of the unknown, the quenching rate constant will decrease abruptly and thus the unknown triplet energy can be bracketed between two known energies. These experiments have failed with carotenoid polyenes, apparently because no donor could be found with an energy low enough to attenuate the quenching rate constant.

Recently, we have carried out a series of laser flash photoacoustic experiments on dyad 2 in order to measure the 
thermal energy which accompanies the relaxation of ${ }^{3} \mathrm{C}-\mathrm{P}$ in solution to its ground-state and thereby calculate the energy of the carotenoid lowest triplet-state. Conceptually, the experiment is straightforward. Relaxation of ${ }^{3} \mathrm{C}-\mathrm{P}$ produces heat in the form of a thermal wave in the solution which is detected with a piezoelectric detector. A reference material having the same absorbance as the unknown and having known energy levels is used to calibrate the spectrometer. Because the photoacoustic experiment is a hybrid of spectroscopic and calorimetric phenomena, analysis can only yield the product of the energy of the triplet-state times its quantum yield and, under favorable conditions, the lifetime of the triplet species under study. By using different diiodomethane concentrations in toluene solution it was possible to use the heavy atom effect to change systematically the triplet quantum yield and lifetime so that the photoacoustic signal was recorded as a function of the quantum yield and lifetime. Separate conventional laser flash experiments on each sample gave an independent measure of the triplet quantum yield and lifetime. Good agreement between the triplet lifetimes from laser flash and photoacoustic measurements confirmed that the carotenoid triplet species was indeed the source of the photoacoustic signal. The triplet energy by this method was calculated to be $0.64 \pm 0.04 \mathrm{eV}$.

Although this is a suprisingly low triplet energy, it does confer advantages on the photoprotective system. A triplet energy well below that of any of the chlorophyll (ca. $1.3 \mathrm{eV}$ ) or bacteriochlorophyll (ca. $1 \mathrm{eV}$ ) species in the photosynthetic array sets the thermodynamics so that the flow of triplet energy to the carotenoid trap will be fast and irreversible. It also ensures that the equilibrium ${ }^{3} \mathrm{C}+\mathrm{O}_{2}=\mathrm{C}+{ }^{1} \mathrm{O}_{2}$ lies far to the left.

Although the measurement of triplet energy reported here was for a synthetic carotenoid pigment, the results should be applicable to a generic carotenoid of $c a .11$ conjugated double bonds such as $\beta$-carotene. It is also noted that a tripletenergy of $0.64 \mathrm{eV}$ corresponds to an optical transition originating at $1937 \mathrm{~nm}$, which explains the lack of reports of phosphorescence. Because the energy gap between the ground and triplet-states is rather small, the triplet potential surface must be deeply embedded in the ground-state potential so that nonradiative relaxation would be expected to be fast, limiting the phosphorescence yield. Consistent with this view, the short triplet lifetime of carotenoid pigments, which is unchanged even in a frozen glassy matrix, is not characteristic of the usual $\pi, \pi^{*}$ triplet-state of dyes and is suggestive of unusually strong coupling to the ground-state.

\section{CAROTENOIDS AND PHOTOMEDICINE: TUMOR DETECTION.}

Aspects of the photoprotective strategy discussed above are relevant to the design of photoactive drugs. It is well known that certain porphyrins localize in some malignant tumor tissues and, upon illumination with light of the proper wavelength, generate powerful oxidizing agents which are toxic to the tumor tissue (refs. 26-28). This tissue damage almost certainly results from the sensitized formation of singlet oxygen by the porphyrin triplet-state. The use of porphyrins in this way is called photodynamic therapy.

From a different perspective, the strongly fluorescent porphyrins are also potentially useful agents for detection and imaging of tumor tissue. The fluorescence contrast between the higher porphyrin concentration in the tumor and the lower level in the surrounding healthy cells can accurately delineate the boundary between healthy and tumor tissue. However, the diagnostic application of tumor-localizing porphyrins is severely limited by the fact that some of the porphyrins or their degradation products remain in other tissues, especially skin. Ilumination of a patient by natural, environmental or diagnostic light results in skin sensitization leading to erythema, serious inflammation and necrosis. Although these problems are a managable side effect in cancer treatment protocols, they are a severe obstacle to screening for early tumor detection. Skin sensitization persists for as long as six to seven weeks until the drug is completely eliminated from the body.

Our knowledge of the photoprotective mechanism suggests a solution to this problem. In carotenoporphyrins such as 2, the carotenoid completely suppresses the formation of singlet oxygen, but has little effect on the porphyrin singlet-state properties, including fluorescence. By eliminating photosensitization problems arising from the porphyrin triplet-state, a tumor-localizing carotenoporphyrin could function well as a diagnostic agent. In fact, carotenoporphyrin 7 (a derivative of 2 in which the meso-tolyl groups are replaced by para-methoxyphenyl groups) functions in just this manner.

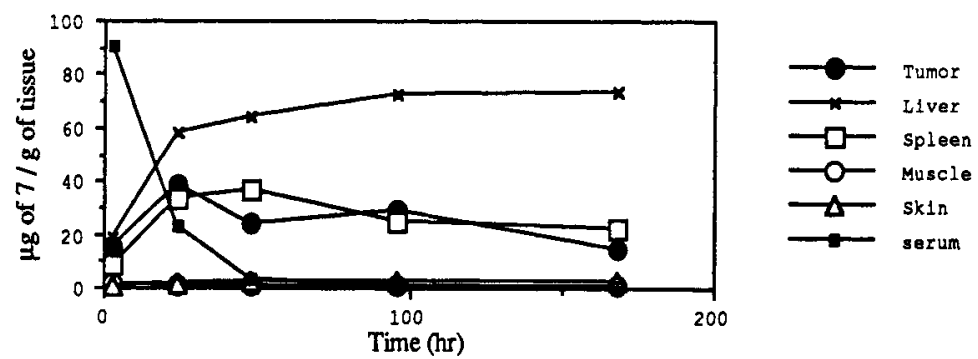

Fig. 1. Carotenoporphyrin distribution in selected tissues as a function of time after intravenous injection of $10.4 \mathrm{mg} / \mathrm{kg}$ of $7 \mathrm{in}$ Cremophor EL. 
In vivo experiments with 7 were carried out with mice bearing an MS-2 fibrosarcoma infiltrating tumor. The carotenoporphyrin was carried by unilamellar liposomes or as an emulsion (Cremophor EL) and intravenously injected. At fixed times after injection, various tissues were extracted and the carotenoporphyrin concentration was measured by absorption and fluorescence techniques. Typical results are shown in Fig. 1. It is clear that by $24 \mathrm{hr}$ after injection, a considerable amount of the carotenoporphyrin was localized in the tumor while relatively small amounts were present in the surrounding muscle and skin tissue. Fluorescence from the localized porphyrin can be readily detected by eye and approximate calculations indicate that with sensitive photomultiplier-based detectors a concentration of a few transformed cells could be detected. Most importantly, experiments with $\mathrm{Balb} / \mathrm{c}$ mice under visible irradiation showed no indication of skin sensitization. It can be noted from Fig. 1 that the carotenoporphyrin localizes in the liver and spleen as well, and elimination from these tissues is slow. We are attempting to reduce or eliminate this problem by altering the hydrophobicity of the carotenoid and porphyrin components.

\section{CAROTENOIDS IN ANIMAL AND PLANT COLORATION}

Carotenoid polyenes are the coloring pigments of a wide range of plants and animals. At high concentrations they act as filters to screen out selected wavelengths of light both to protect light-sensitive tissue and to discriminate between different wavelengths (ref. 29). Carotenoids are poor singlet energy donors to all pigments, not just chlorophylls, and are thus relatively unlikely to serve as sensitizers for the singlet-states of other pigments which may be harmful to a given organism. Because of their very short lifetime, carotenoid singlet-states are unlikely to carry out photochemistry based on diffusional encounters with substrate molecules. Interestingly, carotenoids form aggregates that have highly altered absorption characteristics and thereby provide a wide variety of colors that are a function of the aggregationstate. A good example of this is that astaxanthin in many different pigment-pigment and pigment-protein interactions provides absorption that spans the visible region in the crustacean carapace (ref. 30).

Since the atmosphere became oxidizing ca. 3.2 billion years ago, the suppression of singlet oxygen sensitization has been obligatory for any pigment system. Carotenoids are ideal for coloration in this respect because their triplet species do not sensitize singlet oxygen. Often these pigments occur with others, such as anthocyanins and related flavonoids (ref. 31). It is not known to what extent the carotenoids protect against singlet oxygen damage in these systems. Other strategies for avoiding singlet oxygen sensitization are available. For example, many organisms achieve brilliant coloration by assembling iridescent structures using closely spaced layers of melanin-rich material (refs. 32,33). By occurring in an aggregated-state in vivo, dyes may not sensitize singlet oxygen even though in monomeric form they are potent sensitizers. Presumably, the triplet-states of aggregates either do not form in appreciable yield or are quenched to a very short lifetime and are therefore not kinetically competent for sensitization. Interestingly, a short triplet lifetime of the heme group in hemoglobin is vital for its function as an oxygen carrier, as singlet oxygen sensitization must be precluded. The ferrous ion enhances intersystem crossing in the porphyrin so that its triplet lifetime is only a few nanoseconds. It appears that a common denominator for photoprotection by carotenoids, ferrous ions or aggregation is the quenching of the sensitizer triplet species on a time scale that precludes collisions with dioxygen.

\section{NEWLY DISCOVERED CAROTENOID PHOTOCHEMISTRY}

Two new photochemical reactions of carotenoids have been observed. In one case a carotenoid bearing a terminal anilino or acetanilido group was incorporated into a monolayer and deposited on an indium-tin-oxide electrode. Modulated irradiation of this electrode immersed in a solution containing a redox relay and a counter electrode produced a photocurrent that had an action spectrum very much like the carotenoid absorption spectrum. The polarity of the photocurrent was consistent with electron flow initiated by a carotenoid charge-transfer state. Even though nitrogen-containing carotenoids are not known to occur naturally, an intramolecular charge-transfer state of this compound could mimic the very large excited-state dipole moment reported for certain carotenoid-binding proteins (ref. 34). We have also observed large transmembrane photocurrents in experiments involving planar bilayer lipid membranes doped with the same carotenoids (ref. 35). These results demonstrate that carotenoids can participate in photoinitiated transmembrane signaling and should be regarded as candidates for blue light photoreceptors (ref. 3 ). Very recently, we have observed photoinitiated electron transfer in a carotenoid-porphyrin-diquinone tetrad of the form C-P-Q-Q, (ref. 36), in which the carotenoid excited-state donates an electron to the acceptors to yield ultimately $\mathrm{C}^{+}+\mathrm{P}-\mathrm{Q}-\mathrm{Q}^{--}$. These results illustrate that a pigment with an intrinsically short-lived singlet-state can participate in photochemistry provided that diffusion is not relied upon to bring the reactants together. This implies that, in vivo, pigment-protein complexes that adjust the proximity of the carotenoid to its partners would be required to achieve energy-, and electron-transfer and possibly other photochemical reactions as well.

In conclusion, the carotenoid genre of pigments adapt to photobiological roles with chameleon ease. Their photochemical inertmess and strong absorption are ideal for cryptic coloration of living things. The unusual properties 
of their singlet and triplet-states are uniquely matched to the requirements of anaerobic and oxygen-evolving photosynthesis. When tightly coupled to membrane-linked chemical systems, carotenoids demonstrate photoelectrochemical reactions that make them attractive photoreceptor candidates.

\section{ACKNOWLEDGEMENT}

The authors gratefully acknowledge Hoffmann-LaRoche for providing various carotenoids. This work was supported by grants from the Division of Chemical Sciences, Office of Basic Energy Sciences, Office of Energy Research, U. S. Department of Energy (DE-FG0287ER13791), and the National Science Foundation (CHE-8903216). Acknowledgement is made to the donors of the Petroleum Research Fund, administered by the American Chemical Society, for partial support of this research. This is publication number 157 from the Center for the Study of Early Events in Photosynthesis at Arizona State University.

\section{REFERENCES}

1. R. J. Cogdell and H. A. Frank, Biochim. Biophys. Acta, 895, 63-79 (1987).

2. B. Demmig-Adams, Biochim. Biophys. Acta, 1020, 1-24 (1990).

3. A. Srevastava and E. Zeiger, Plant Physiol., 100, 1562-1566 (1992).

4. T. G. Truscott, J. Photochem. Photobiol. 6, 359-371 (1990).

5. D. Gust, T. A. Moore and A. L. Moore, Acc. Chem. Res. 26, 198-205 (1993).

6. D. Gust, T. A. Moore, A. L. Moore, J. Jori, and E. Reddi, Ann. N. Y. Acad. Sci., in press.

7. R. M. Hermant, P. A. Liddell, S. Lin, R. G. Alden, H. K. Kang, A. L. Moore, T. A. Moore and D. Gust, J. Am. Chem. Soc., $115,2080-2081$ (1993).

8. P. F. Jones, W. J. Jones and B. H. Davies, J. Photochem. Photobiol. A: Chem. 68, 59-75, (1992).

9. M. Mimuro, U. Nagashima, S. Nagaoka, S. Takaichi, I. Yamazaki, Y. Nishimura and T. Katoh, Chem. Phys. Letters, 204, 101105, (1993).

10. T. Gillbro, P. O. Andersson, R. H. Liu, A. E. Asato, S. Takaichi and R. J. Cogdell, Photochem. Photobiol.,57, 44-48 (1993).

11. Y. Watanabe, T. Kameyama, Y. Miki, M. Kuki, and Y. Koyama, Chem. Phys. Letters, 206, 62-68 (1993).

12. H. A. Frank, R. Farhoosh, R. Gebhard, J. Lugtenburg, D. Gosztola, and M. R. Wasielewski, Chem. Phys. Letters, 207, 8892 (1993).

13. M. R. Wasielewski and L. D. Kispert, Chem. Phys. Letters, 128, 238-243 (1986).

14. J. K. Trautman, A. P. Shreve, T. G. Owens, and A. C. Albrecht, Chem. Phys. Letters, 166, 369-374 (1990).

15. K. Jorgensen, H. Stapelfeldt, and L. H. Skibsted, Chem. Phys. Letters, 190, 514-519 (1992).

16. T. Gillbro and R. J. Cogdell, Chem. Phys. Letters, 158, 312-316 (1989).

17. D. Gust, T. A. Moore, A. L. Moore, F. Gao, D. Luttrull, J. M. DeGraziano, X. C. Ma, L. R. Makings, S.-J. Lee, T. T. Trier, E. Bittersmann, G. R. Seely, S. Woodward, R. V. Bensasson, M. Rougée, F. C. De Schryver, and M. Van der Auweraer, $J$. Am. Chem. Soc., 113, 3638-3649 (1991).

18. D. Gust, T. A. Moore, A. L. Moore, C. Devadoss, P. A. Liddell, R. Hermant, R. A. Nieman, L. J. Demanche, J.M. DeGraziano, and I. Gouni, J. Am. Chem. Soc., 114,3590-3603 (1992).

19. T. A. Moore, D. Gust and A. L. Moore, in Carotenoids Chemistry and Biology, (N. I. Krinsky, M. M. Mathews-Roth and R. F. Taylor, eds), pp. 223-228, Plenum Press, N. Y. (1989).

20. P. A. Liddell, D. Barrett, L. R. Makings, P. J. Pessiki, D. Gust, and T. A. Moore, J. Am. Chem. Soc., 108, 5350-5352 (1986).

21. M. R. Wasielewski, P. A. Liddell, D. Barrett, T. A. Moore and D. Gust, Nature, 322, 570-572 (1986),

22. R. J. Cogdell, T. G. Monger and W. W. Parson, Biochim. Biophys, Acta, 408, 189-199 (1975); C. C. Schenck, P. Mathis and M. Lutz, Photochem. Photobiol., 39, 407-417 (1984).

23. T. O. Yeats, H. Komiya, A. Chirino, D. C. Rees, J. P. Allen, and G. Feher, Proc. Natl. Acad. Sci. USA, 85, 7993-7997 (1988).

24. D. Gust, T. A. Moore, A. L. Moore, A. Krasnovsky, Jr., P. A. Liddell, D. Nicodem, J. M. De Graziano, P. Kerrigan, L. R. Makings, and P. Pessiki, J. Am. Chem. Soc. 115 in press (1993).

25. Unpublished experiments performed in collaboration with $S$. Boxer.

26. J. Moan and K. Berg, Photochem. Photobiol. 55, 931-948 (1992).

27. B. W. Henderson and T. J. Dougherty, Photochem. Photobiol. 55, 145-157, (1992).

28. D. Kessel, in Photodynamic Therapy of Neoplastic Disease. (D. Kessel, ed) Vol. II: pp. 1-13. CRC Press, Boca Raton, FL. (1990)

29. P. A. Liebman and A. M. Granda, Nature, 253, 370-372 (1975).

30. M. L. Mackenthun, R. D. Tom and T. A Moore, Nature, 279, 265-266, (1979), and original references therein.

31. C. H. Eugster and E. Märki-Fischer, Angew. Chem. Int. Ed. Engl., 30, 654-672 (1991).

32. D. S. Fox, Animal Structural Colors and Biochromes, (University of California Press, 1978)

33. D. W. Lee, Nature, 349, 260-262 (1991).

34. D. S. Gottfried, M. A. Steffen, and S. G. Boxer, Science, 251, 662-665 (1991).

35. A. L. Moore, D. Gust, T. A. Moore, L. Sereno, J. J. Silber, P. A. Liddell, P. Seta, and E. Bienvenue, Photochem. Photobiol., $55 S, 101 S$ (1992).

36. S.-J.Lee, J. M. DeGraziano, A. N. Macpherson, E.-J. Shin, P. K. Kerrigan, G. R. Seely, A. L. Moore, T. A. Moore and D. Gust, Chem. Phys. in press, 1993. 